hep-ph/0010312

\title{
WHAT WILL WE LEARN IF A HIGGS BOSON IS FOUND?
}

\author{
G. L. Kane ${ }^{1}$, S. F. King ${ }^{2}$ and Lian-Tao Wang ${ }^{1}$ \\ ${ }^{1}$ Randall Physics Laboratory, University of Michigan, Ann Arbor, MI 48109-1120 \\ ${ }^{2}$ Department of Physics and Astronomy, University of Southampton, Southampton, \\ SO17 1BJ, U.K.
}

\begin{abstract}
We examine what can be deduced from the discovery of a Higgs boson, focusing on a possible discovery at LEP. The analysis begins with the most general situation where no further constraints can be deduced, and then specializes to include various assumptions. Assuming naturalness, the relatively large mass suggests that one or more of larger $\tan \beta$, large phase, or extra singlets is present. We discuss the implications of a Higgs mass discovery for the SUSY spectrum, and in particular gluinos and stops. We show that a lighter gluino produceable at the Tevatron remains a likely possibility, in disagreement with a recent claim.
\end{abstract}

November 12, 2018 


\section{Introduction}

The discovery of a Higgs boson will be of profound importance. Arguably, it is one of the most important experimental results of all time. The Higgs boson is a new kind of matter, the first kind to be found in a century. New quarks and leptons have been found, but they are all particles like the electron that carry other charges. New force-mediating bosons have been found but they are all quanta like the photon. The Higgs boson is the quantum of a new kind of field, one for which the energy density of the universe is lower when the field has a non-zero value, and one whose interactions with other particles (both bosons and fermions) allow them to have mass. The Higgs boson both completes the Standard Model of particle physics, and points to how to extend the Standard Model. This discovery will also be a remarkable achievement for human intelligence, which was able to generate the idea of such a particle in nature and then to construct experimental facilities in which it could be produced and detected. It will lead us into a new realm of understanding the laws of nature.

In view of the profound importance of the Higgs discovery the present paper is designed to be largely accessible to the non-specialist. The purpose of the paper is to discuss what the measured values of mass and production rate (assuming a discovery) can tell us about the parameters of the Higgs sector, and about how the Standard Model (SM) will be extended. Many of the points in this paper will hold whether a Higgs boson is found at LEP or at Fermilab. However, we will focus on the possibility that the discovery occurs at LEP, and in that case the mass is already approximately known (since the collider is running at or near its highest energy), and the cross section times branching ratio must be as large as possible to get enough events to 
observe them. Initially, confirmation that a discovery is indeed a Higgs boson will rely on seeing a decay dominated by couplings proportional to mass, i.e., mainly to $b \bar{b}$, with a $\tau \bar{\tau}$ branching ratio (at about $8 \%$ ) observed later, plus observing the expected production rate approximately. One important question is to identify whether the observed Higgs boson is that of the SM, a light Higgs boson of the supersymmetric (SUSY) Higgs spectrum, or a composite state. If the mass of the Higgs boson is about $115 \mathrm{GeV}$, one can argue that it is not the SM Higgs boson, because it is well below the lower limit (of about $130 \mathrm{GeV}$ ) that destabilizes the vacuum [1]. Further, while in composite approaches to the origin of mass one cannot formulate theories well enough to make precise predictions, the natural mass for a scalar in such theories is near the non-perturbative limit. (For example, ref [2] gives an approximate region $165<m_{h}<230 \mathrm{GeV}$, given certain assumptions.) Thus it is very unlikely that a discovery at LEP could be interpreted as a composite Higgs. In contrast there is motivation to study the case of a SUSY Higgs boson for several reasons. It is well known that the mass of a light scalar is not protected against radiative corrections that raise it up to the unification or Planck scale except in the presence of a symmetry such as SUSY. In addition SUSY predicts a Higgs boson mass consistent with a LEP measurement. Therefore we presume that such a discovery would be the lightest Higgs boson of the supersymmetric theory and proceed to study it in that context. However if the Higgs boson is indeed found at LEP the mass and cross section do not allow us to prove from its properties that it is explicitly a supersymmetric Higgs boson [3]. Direct evidence for SUSY will have to come from discovery of superpartners or of some of the additional Higgs bosons at LEP, Fermilab or LHC. Less direct evidence for SUSY could come from observed deviations of the Higgs boson properties from the SM values when more precise data is available. 
It is known that in the general case the number of parameters needed to describe the Higgs sector in SUSY models is at least seven [4, 5, 6, 7. At tree level the number is only two, but (as was first pointed out in Ref. (⿴囗十) the rest enter via large top-stop loop contributions to the Higgs potential that can shift the mass and cross section and branching ratios significantly. If $\tan \beta$, the ratio of Higgs vacuum expectation values, is large, or if the theory is an extension of the minimal supersymmetric SM (MSSM), there are surely more parameters (from b- $\tilde{b}$ loops, or from the mixing of additional scalars). Other loop contributions such as W-chargino and charged Higgs loops can be important as well (see for example [8] ). If the mass and cross section are measured there are two relations among the seven or more parameters, and without further input there are unfortunately no additional results that can be deduced, apart from the general results of the above two paragraphs. It should also be noted that $115 \mathrm{GeV}$ is pushing the constraints on allowing electroweak baryogenesis in the MSSM [9], though those limits are soft ones.

Perhaps the most important success of supersymmetry is that it provides a radiative mechanism by which the breaking of the electroweak $(\mathrm{SU}(2) \times \mathrm{U}(1))$ symmetry can be explained. If radiative electroweak symmetry breaking is indeed correct, it leads to a relation between the measured $\mathrm{W}$ or $\mathrm{Z}$ masses and the soft supersymmetry breaking masses. If this relation is indeed physics, it must not involve fine-tuned differences of large numbers. If we assume this is so then we get important constraints on the parameter space. This is often called the naturalness assumption [10, 11]. This assumption can be checked a posteriori since it implies the production of gluinos at the Tevatron, and has further implications that we describe below.

Given that a LEP Higgs boson suggests a state with full strength couplings to W, 
$\mathrm{Z}$ and small invisible branching ratio, we can usefully estimate the number of Higgs bosons that will be produced at the Tevatron. Confirmation of a signal in a given mass bin will require less data than a discovery. Once the Higgs mass is measured (e.g. at LEP), all the Higgs bosons produced at the Tevatron [12] (even the inclusive channel) can be studied to confirm the signal and to observe smaller decay branching ratios, etc. The expected number is of order 15,000 per $10 \mathrm{fb}^{-1}$. If the Tevatron collider and detectors achieve design luminosity and are not limited by funding shortages, they will gather $\sim 30 \mathrm{fb}^{-1}$. Thus with a known mass we can confirm a signal and even hope to see even $B R(\gamma \gamma)$ (which is expected to be $\sim 10^{-3}$.) Observing the $\gamma \gamma$ decay immediately proves that $h$ does not have spin 1 , and the value of the branching ratio is a useful constraint on other new particles in the theory.

The mass of $115 \mathrm{GeV}$ is actually somewhat large for what is expected in the minimal theory if we insist it is not unnatural [11, 13]. The full parameter space of the MSSM allows masses up to about $130 \mathrm{GeV}$, but if the soft phases are zero the region above about $105 \mathrm{GeV}$ is essentially excluded by naturalness unless $\tan \beta$ is rather large, in which case masses up to about $115 \mathrm{GeV}$ are allowed. Thus the LEP mass could be evidence that $\tan \beta$ is large. Alternatively, with general phases one can have $m_{h}=115 \mathrm{GeV}$ for smaller $\tan \beta$, so the LEP mass could suggest large phases. Also, when an extra $\mathrm{SU}(2)$ singlet scalar is added to the theory the upper limit on the mass for a given $\tan \beta$ and naturalness increases about $30 \mathrm{GeV}$ [14, 15], so the larger mass could also be evidence for this extension of the MSSM. Of course, a mixture of these effects could be present, or even more general extensions of the MSSM are possible.

When the full SUSY parameter space is present (including the phase which enters 
the Higgs potential), one might wonder if the production rate can be larger than in the SM. The production cross section can never increase for any parameter over the SM one, but the $\mathrm{BR}(b \bar{b})$ can be larger than the SM one for special parameters [16] if the branching ratios to other channels are suppressed. This can at most give about a $15 \%$ increase in cross section times branching ratio.

Another issue that needs to be studied is the possibility that the "observed" scalar is actually the second lightest one, the lightest being largely decoupled. The arises over a significant region of the parameter space of the theories with extra scalars [15]. This effect can also happen in the MSSM in the presence of phases (a suggestion to that effect has appeared in Ref [6] as this paper was being written.)

In the following, we first discuss the general situation and then specialize to increasingly specific approaches to the Higgs sector.

\section{General Case}

The most general SUSY model may involve an explicit Higgs mass $\mu$, plus a rather general scalar sector, and involve many complex parameters. In such a framework one can deduce no further implications from the measurement of the Higgs mass and production. With rotations one can essentially consider here the two usual Higgs doublets $H_{U}$ and $H_{D}$ plus a single gauge singlet $N$ whose vacuum expectation value is responsible for generating the $\mu$ term due to the coupling $\lambda N H_{U} H_{D}$. In a recent analysis (which did not include the effect of phases) one of us showed that in the NMSSM the low $\tan \beta$ region is technically natural and permitted a strong first order phase transition consistent with electroweak baryogenesis [17]. The reason for the improved 
naturalness of the NMSSM may be simply understood since the tree-level Higgs mass squared formula is modified from its MSSM value to $M_{Z}^{2}\left(\cos ^{2} 2 \beta+C \sin ^{2} 2 \beta\right)$ where $C=2 \lambda^{2} /\left(g^{2}+g^{\prime 2}\right)$ [14. As we shall see later an increased tree-level Higgs mass demands less from radiative corrections. The extra Higgs singlet state may mix with or decouple from the physical Higgs state, leading to possible interesting effects.

Specializing to the MSSM, with an explicit Higgs mass term $\mu H_{U} H_{D}$, it was first recognized a couple of years ago that the Higgs parameter space had been oversimplified [4, 5, 6, 7] and that some of the parameters could be complex. This has now been studied in some detail [6, 7], [18]- 22, and possible effects are large. All the extra parameters enter from loop diagrams that contribute significantly to the Higgs potential. The Higgs mass eigenstates and their couplings to gauge bosons and fermions can be modified. Any conclusion about the Higgs sector - limits or implications of discoveries — that are made without including the full parameter space are not justified and may be simply wrong [7]. The top-stop loop is presumably the dominant one and including it one can list the minimum set of parameters on which the Higgs potential (and hence Higgs mass and couplings) depends as $M_{L}^{2}, M_{R}^{2},\left|A_{t}\right|,|\mu|$, $\phi_{\mu}+\phi_{A_{t}}, B=m_{3}^{3}, \tan \beta$. The first three are the left and right handed stop soft breaking square masses and the stop trilinear soft mass parameter, then the Higgs mass parameter $\mu$, followed by the phase combination that arises as the sum of the phase of $\mu$ and the phase of $A_{t}$. B is the Higgs mixing parameter in the soft Lagrangian. Finally there is $\tan \beta$. The Higgs mass is directly measured kinematically if there is a signal at LEP. Similarly, if there is a signal the cross section is about equal to the SM one, which implies that the masses of the heavier Higgs bosons are large and they effectively decouple; this eliminates one combination of parameters. Thus there are five parameters left. If $\tan \beta$ is large there is an important bottom-sbottom loop too, 
with more parameters, and chargino and neutralino and charged Higgs loops could also be non-negligible [8]. In the latter cases there are too many parameters and it is impossible to learn anything beyond the direct data and the implications outlined in the introduction.

However, we think it is worthwhile to proceed with simplifying assumptions below and see what can be deduced if we make some assumptions. While the assumptions may be right, they may not, and the results should not be believed just because otherwise we cannot learn anything - that is "lamppost physics". Rather, as we will see, at least to some extent the assumptions can be checked a posteriori with more data. For all cases we consider, we can point out what analyses are useful, but final studies and implications have to be done by experimenters who can include efficiencies and errors in appropriate ways.

\section{$3 \quad$ Naturalness}

We think the safest assumption is that if nature is indeed supersymmetric at the weak scale, and this provides the actual explanation of EWSB, then this explanation will be natural, as discussed above. In that case we can study the general parameter space and find that it is indeed somewhat reduced in size. Some lower values of $\tan \beta$ are excluded, but only up to $\tan \beta$ of about 3-4. The phase remains general, unfortunately, with any value allowed. The reduction of the parameter space can be seen most clearly in the $\mu$ parameter, which has an upper limit of about $300 \mathrm{GeV}$. This limit is not very sensitive to other parameters, and not too sensitive to the

unknown phase. It has considerable implications, particular for the neutralino and chargino masses, for $b \rightarrow s \gamma$ branching ratio and CP asymmetry, and for the LSP 
cold dark matter relic density and detectability.

In the context of naturalness one may wonder about the question of phases in SUSY. If all the phases are large, there must be some cancellations responsible for the smallness of the neutron and electron electric dipole moments (EDMs) [23], and given our present understanding this cancellation mechanism apparently violates naturalness. However the cancellations required are model dependent, and in some theories this mechanism could be entirely natural. Another possibility is that the first two families of squarks and sleptons are very heavy, while the third family ones are lighter; then the Higgs potential is unchanged, but the contribution to EDMs is reduced, so the appeal to cancellations is reduced. In this case the needed mass hierarchy would have to be explained. Alternatively there may be small SUSY phases for some presently unknown theoretical reason. It is to this possibility that we now turn.

\section{Small Phases, Large $M_{A}$ and Larger $\tan \beta$.}

From now on we set the phase in the Higgs potential to zero, so CP is conserved, and also assume that $B$ is rather large (which physically means a large CP-odd peudoscalar mass $\left.M_{A}>M_{Z}\right)$ since it is known that in this limit the Higgs production crosssection becomes maximal, consistent with a LEP observation. In this case the Higgs potential only depends on the four parameters $M_{L}^{2}, M_{R}^{2}, M_{L R}^{2}=m_{t}\left(A_{t}-\mu \cot \beta\right)$, $\tan \beta$, where $B$ has decoupled, and the phase has disappeared leaving only the relative sign of $A_{t}$ and $\mu$ appearing in the combination $M_{L R}^{2}$. The first three of these parameters completely determine the physical stop masses $m_{1}, m_{2}$ and stop mixing angle $\theta_{t}$. Another important question to address is the sign of $\mu$. In the Higgs production process we are considering, only the relative sign between $\mu$ and the trilinear coupling $A_{t}$ is relevant. We found that for any set of parameter with one relative sign ('+' for 
example) that is consistent with the signal, there is another point in the parameter space with opposite sign that is allowed. Therefore, we cannot decide the relative sign if we take the full parameter space into consideration.

Given these assumptions (including naturalness) a corollary is that $\tan \beta$ is larger, say 5-20, the upper limit chosen to avoid the complications of the bottom quarks and squarks. Naturalness prefers larger $\tan \beta$ for two independent reasons: the tree-level contribution to the Higgs mass $M_{Z} \cos 2 \beta$ is maximised; and the top quark Yukawa coupling is reduced (see later).

Assuming larger $\tan \beta$ the tree-level Higgs mass is near $M_{Z}$. Can one make any statements about the stop spectrum from the condition that its radiative corrections shift the Higgs mass from $M_{Z}$ to about $115 \mathrm{GeV}$, a difference of about $25 \mathrm{GeV}$ ? There are two dominant radiative corrections from the stop sector, a "degenerate" contribution depending on $\ln \left(m_{1}^{2} m_{2}^{2} / m_{t}^{4}\right)$, and a "hierarchical" contribution depending on $\ln \left(m_{2}^{2} / m_{1}^{2}\right)$ and proportional to the combination $\sin ^{2} 2 \theta_{t}\left(m_{2}^{2}-m_{1}^{2}\right) / 2 m_{t}^{2}$. It is possible to obtain the required radiative corrections from either a degenerate heavy stop spectrum with a small mixing angle, or a hierarchical stop spectrum with a large mixing angle and a light stop squark. Degenerate stop squarks with large mixing is also possible. There is no way to choose among them without further information. Thus from the point of view of the low energy theory it is difficult to learn much about the stop spectrum from the Higgs mass, although once one stop mass is measured the remaining stop mass and mixing angle will be constrained.

\section{$5 \quad$ High Energy Input Parameters}

We now explicitly assume that the low energy parameters such as the stop parameters and Higgs soft masses are determined from some input parameters at a high energy 
scale, which for definiteness may be taken to be the unification scale $10^{16} \mathrm{GeV}$. The relevant high energy input parameters are $M_{i}(0), m_{Q}(0), m_{U}(0), m_{H_{U}}(0), m_{H_{D}}(0), A(0)$ (the high scale gaugino soft masses, squark doublet soft mass, stop singlet soft mass, Higgs soft masses, and trilinear soft mass, respectively), together with $\operatorname{sign}(\mu)$ and $\tan \beta$, where $|\mu|$ and $B$ are fixed by the low electroweak symmetry breaking conditions (unlike the previous approach where the low energy Higgs soft masses were fixed by these conditions.)

The radiative symmetry breaking mechanism involves the high energy Higgs soft mass squared $m_{H_{U}}^{2}(0)$ being positive but the low energy value $m_{H_{U}}^{2}(t)$, where $t=\ln Q$ and $Q$ is the low energy $\overline{M S}$ scale, having been driven negative. Naturalness requires that $\left|m_{H_{U}}^{2}(t)\right|$ should not be much larger than $M_{Z}^{2}$, and in order to prevent this we require $m_{H_{U}}^{2}(t)$ not to be too negative. As mentioned larger $\tan \beta$ implies a smaller top Yukawa coupling which helps not to drive $m_{H_{U}}^{2}(t)$ too negative. Another requirement is that the high energy gluino mass $M_{3}(0)$ not be too large, since large gluino mass drives the squark mass squareds $m_{Q}^{2}(t), m_{U}^{2}(t)$ very positive, and the coupled renormalisation group equations drive $m_{H_{U}}^{2}(t)$ very negative in response. However $m_{Q}^{2}(t), m_{U}^{2}(t)$ are essentially just the stop parameters $M_{L}^{2}, M_{R}^{2}$ and the 115 $\mathrm{GeV}$ Higgs mass requires at least one of them to be quite large, as discussed, so subtlety is required to avoid some violation of naive naturalness [11].

Implications for the SUSY spectrum of a 114-115 GeV Higgs have recently been explored assuming the minimal SUGRA relations $M_{1 / 2}=M_{i}(0)$ and $m_{0}=m_{Q}(0)=$ $m_{U}(0)=m_{H_{U}}(0)=m_{H_{D}}(0)$ [26], and it was concluded there that $M_{1 / 2}>250 \mathrm{GeV}$. However we note that these authors restrict themselves to small values of $m_{0} \leq 200$ $\mathrm{GeV}$ where the cosmological relic density calculated with their assumptions is in the desired range. In our view, the implications of knowing $m_{h}$ for cold dark matter can be 
summarized as follows. While we do expect the LSP to be the lightest neutralino, and to be a significant portion of the cold dark matter, the actual calculation of the relic density is known to be very sensitive to a variety of assumptions. For example, $\Omega_{L S P} \sim$ $1 / 3$ can be the result of calculations with lighter sleptons but heavier squarks once one does not insist on a single scalar mass $m_{0}$. Or the presence of phases can lead to $\Omega_{L S P} \sim 1 / 3$ [27] when it would not if the phases were zero. Or most of the relic density can be created in non-thermal processes [28]. While the actual value of $m_{h}$ does enter all calculations, we do not think it leads to any significant increase in understanding the CDM relic density at the present time, because numerical results require too many major assumptions. In particular we find the assumptions of minimal SUGRA and $m_{0}<200 \mathrm{GeV}$ to be unnecessarily restrictive.

As an illustrative point, we find that for larger values of $\tan \beta$ and taking $m_{0}=500$ $\mathrm{GeV}$ with $A(0)=-2 m_{0}$ (in our convention above) that the $M_{1 / 2}$ required for a 114 $115 \mathrm{GeV}$ Higgs is reduced to about $190 \mathrm{GeV}$ for positive $\mu$ (the Higgs measurement does not constrain the sign of $\mu$ ), with a significantly lighter stop mass of about 240 $\mathrm{GeV}$ and a chargino mass of about $130 \mathrm{GeV}$. If in addition we allow a slight violation of scalar universality by increasing $m_{H_{U}}(0)$ by $25 \%$ relative to the other scalar masses then $M_{1 / 2}$ is reduced still further to about $180 \mathrm{GeV}$, with a reduced stop mass of about $160 \mathrm{GeV}$, and a chargino mass about $140 \mathrm{GeV}$. Maintaining scalar universality but relaxing gaugino mass universality with $M_{2}(0)=2 M_{3}(0)$, and taking $m_{0}=500 \mathrm{GeV}$ and $A(0)=-2 m_{0}$ as before, we find that $M_{3}(0)$ required for a $114-115 \mathrm{GeV}$ Higgs is reduced to $150 \mathrm{GeV}$ with a very light stop mass of $80 \mathrm{GeV}$ but a heavier chargino of mass $240 \mathrm{GeV}$. The effects of non-universality can be understood as resulting from changes in the $\mu$ parameter due to changes in $m_{H_{U}}^{2}(t)$ due to renormalisation group effects. Similar effects can be obtained for $m_{0} \sim 1 \mathrm{TeV}, A(0)>1 \mathrm{TeV}$ but with 
reduced naturalness.

Does naturalness favour a lighter gluino, possibly in the Tevatron range? Given our assumptions we require some large parameter in the stop sector to increase the Higgs mass by $25 \mathrm{GeV}$ due to radiative corrections. The origin of this large parameter may come either directly at the high scale, by for instance choosing $m_{0} \sim 500 \mathrm{GeV}$, $A(0) \sim 1 \mathrm{TeV}$, in which case $M_{3}(0) \sim 150 \mathrm{GeV}$ and the gluino can be lighter.

Or it could arise from having relatively small squark soft parameters at the high scale, but having a large gluino mass which increases the stop parameters through renormalisation group running effects. Both possibilities seem equally likely from the naturalness point of view. The lighter gluino case is testable at the Tevatron. Of course in the more general case with the phase present then the gluino can be lighter than in the zero phase case, for a given Higgs mass and $\tan \beta$. In general when the theory is extended to improve naturalness a lighter gluino will be favoured.

\section{Conclusion}

We have discussed in general terms the implications of a possible Higgs boson discovery. We first argued that a Higgs boson discovery at LEP is evidence for a SUSY Higgs boson. The most general supersymmetry theory is too complicated to draw any further conclusions from a Higgs boson mass measurement. We think it is worthwhile to make a series of assumptions and examine the consequences at each stage. We first considered the NMSSM (which extends the minimal supersymmetric theory to include an additional scalar) where naturalness can be improved relative to the MSSM for low $\tan \beta$ and electroweak baryogenesis looks more promising. Turning to the MSSM, we emphasised that the presence of phases can lead to $\tan \beta$ being lower than expected for a given Higgs mass. The $\mathrm{BR}(b \bar{b})$ can be larger than the SM one 
for special parameters [16] if the branching ratios to other channels are suppressed, allowing at most about a $15 \%$ increase in cross section times branching ratio. We then imposed naturalness where an upper limit on $\mu$ could be set. Setting phases to zero and assuming large $M_{A}$ reduces the number of parameters considerably and with those assumptions naturalness probably implies larger $\tan \beta$. The stop spectrum must satisfy the constraint of increasing the Higgs mass by about $25 \mathrm{GeV}$; it can do this in several ways. We then considered the high energy input parameters and showed that with these assumptions some subtlety is required to preserve naturalness. We concluded that a lighter gluino produceable at the Tevatron is likely when the theory is extended to improve naturalness, and will coincide with a lighter stop squark, since both effects arise from a larger trilinear soft mass. This conclusion disagrees with a recent result due to the unnecessarily restrictive nature of the assumptions in that case.

Finally it is still necessary to consider the possibility that LEP will only set a bound on the Higgs mass, and not make a discovery, in which case the true supersymmetric Higgs mass limit is well below the LEP kinematic limit. In this case the cross section is not saturated, and the true limit has to be deduced from the full

parameter space. For the mass, the limit is no longer about $115 \mathrm{GeV}$, but is instead about $20 \%$ lower, about $95 \mathrm{GeV}$ [7, 6. No discovery does not yet imply a conflict with naturalness, since in the general parameter space a given cross section can occur for a lower mass.

\section{Acknowledgment}

G.K. thanks A.Schwimmer for a question. We are very grateful to L.Everett for 
discussions and comments on the paper, and to S.Rigolin for a read of the paper. S.K. is grateful to the Randall Physics Laboratory at the University of Michigan and the Aspen Center for Physics where some of this work was done, and to PPARC for the support of a Senior Fellowship.

\section{References}

[1] For a review, see M.Sher Phys. Rep.179 273, 1989. Recent calculation can be traced from J.R. Espinosa and M. Quiros, Phys.Rev.Lett.81:516,1998, hep$\mathrm{ph} / 9809269$.

[2] N. Arkani-Hamed, H.-. Cheng, B.A. Dobrescu, L.J. Hall, hep-ph/0006238.

[3] The following paper studies how to deduce general properties of new scalar particles: C.P.Burgess, J.Matias and M.Pospelov, hep-ph/9912459.

[4] M. Brhlik and G.L. Kane, Phys.Lett.B437:331,1998, hep-ph/9803391.

[5] A. Pilaftsis, Phys. Lett. B435:88, 1998, hep-ph/9805373; Phys. Rev. D58:096010, 1998, hep-ph/9803297.

[6] A. Pilaftsis,C.E.M. Wagner, Nucl.Phys.B553:3, 1999, hep-ph/9902371; M. Carena, J. Ellis, A. Pilaftsis, C.E.M. Wagner, hep-ph/0009212.

[7] G.L. Kane and L.-T. Wang Phys.Lett.B488:383, 2000, hep-ph/0003198.

[8] T. Ibrahim and P. Nath hep-ph/0008237.

[9] M. Carena, M. Quiros and C.E.M. Wagner Nucl.Phys.B524:3,1998 and earlier papers listed there; M. Brhlik, G.J. Good, G.L. Kane, hep-ph/9911243. 
[10] J. Ellis, K. Enqvist, D. Nanopoulos and F. Zwirner, Nucl. Phys. B 276:14, 1986; R. Barbieri and G.-F. Giudice, Nucl. Phys. B 306:63, 1988; G. W. Anderson and D. J. Castano, Phys. Lett. B 347:300, 1995; G. W. Anderson and D. J. Castano, Phys. Rev. D 52:1693, 1995; G. W. Anderson and D. J. Castano, Phys. Rev. D 53:2403, 1996; G.W. Anderson, D. J. Castano and A. Riotto, Phys. Rev. D 55:2950, 1997; S. Dimopoulos and G.-F. Giudice, Phys.Lett. B 357:573, 1995.

[11] More general treatments of this problem are G.L. Kane and S.F. King Phys.Lett.B451:113, 1999 and M. Bastero-Gil, G.L. Kane and S.F. King Phys.Lett.B474:103, 2000 hep-ph/9910506.

[12] For Tevatron Higgs capabilities see M. Carena et al, hep-ph/0010338.

[13] Another recent expression of the concern about the large $m_{h}$ is R. Barbieri, A. Strumia hep-ph/0005203.

[14] T. Moroi and Y. Okada, Phys.Lett.B295:73, 1992; G. Kane, C. Kolda and J. Wells Phys.Rev.Lett.70:2686, 1993; J.R. Espinosa and M. Quiros, Phys.Lett.B291:115, 1992.

[15] U. Ellwanger, M.R. de Traubenberg, C.A. Savoy Phys.Lett.B315:331, 1993; J. Kamoshita Y. Okada and M. Tanaka, Phys.Lett.B328:67, 1994; T. Elliott, S.F. King and P.L. White, Phys.Rev.D49:2435, 1994; S.F. King and P.L. White, Phys.Rev.D52:4183, 1995; U. Ellwanger, M.R. de Traubenberg, CRN Strasbourg, C.A. Savoy Z.Phys.C67:665, 1995; J.R. Espinosa and J.F. Gunion Phys.Rev.Lett.82:1084, 1999.

[16] K.S. Babu and C. Kolda, Phys.Lett.B451:77, 1999; M. Carena, S. Mrenna, and C.E.M. Wagner, Phys.Rev.D60:075010, 1999. 
[17] M. Bastero-Gil, C. Hugonie, S.F. King, D.P. Roy and S. Vempati, Phys. Lett. B489:359, 2000.

[18] K.S. Babu, C. Kolda, J. March-Russell and F. Wilczek, Phys.Rev.D59:016004, 1999, hep-ph/9804355.

[19] D.A. Demir, Phys.Rev.D60:055006, 1999, hep-ph/9901389.

[20] D.A. Demir, Phys.Lett.B465:177, 1999, hep-ph/9809360.

[21] S.Y. Choi, M. Drees and J.S. Lee, KIAS-P00012, Feb 2000, hep-ph/0002287.

[22] B. Grazdkowski, J. Gunion, and J. Kalinowski, hep-ph/0001093

[23] T. Ibrahim, P. Nath, Phys.Rev.D58:111301, 1998, Erratum-ibid.D60:099902, 1999; Phys.Rev.D57:478, 1998; Phys.Lett.B418:98, 1998; T. Falk, K.A. Olive, Phys.Lett.B439:71, 1998 ; M. Brhlik, G.J. Good, G.L. Kane, Phys.Rev.D59:115004, 1999, hep-ph/9810457; S. Pokorski, J. Rosiek, C.A. Savoy, Nucl.Phys.B570:81, 2000.

[24] M. Carena, P.H. Chankowski, S. Pokorski, C.E.M. Wagner, hep-ph/9805349; J. Casas, J. R. Espinosa and H. Haber, hep-ph/9801365.

[25] H. Haber and R. Hempfling, Phys. Rev. Lett. 66:1815, 1991; Y. Okada, M. Yamaguchi and T. Yanagida, Prog. Theor. Phys. 85:1, 1991; J. Ellis, G. Ridolfi and F. Zwirner, Phys. Lett. B 257:83, 1991; J. Ellis, G. Ridolfi and F. Zwirner, Phys. Lett. B 262:477, 1991.

[26] J. Ellis, G. Ganis, D.V. Nanopoulos and K. Olive, hep-ph/0009355.

[27] M. Brhlik, D.J.H. Chung and G.L. Kane, hep-ph/0005158. 
[28] G.F. Giudice, E.W. Kolb and A. Riotto, hep-ph/0005123; D.J.H. Chung, E.W. Kolb and A. Riotto, Phys.Rev.D60:063504, 1999; M. Bastero-Gil and S.F. King, in preparation. 